\title{
PRACTICAL ASPECTS OF 3D COHERENT NOISE FILTERING USING (F-Kx-Ky) OR WAVELET TRANSFORM FILTERS \\ P.Y.GALIBERT ${ }^{*}$, L. DUVAL ${ }^{2}$, R.DUPONT ${ }^{l}$ \\ ${ }^{1}$ CGG,Paris,France ${ }^{2}$ IFP, Paris, France
}

\section{Summary}

Source generated surface waves are a long-standing issue in land seismic data processing. Indeed they represent one of the main obstacles to the development of time-lapse surveys on land. The present work compares the results of coherent noise filtering obtained on real data sets by using the popular 3D wavenumber filter with the results obtained by using 3D filters designed with wavelet transform techniques. Practical aspects of sampling irregularities are discussed, with respect to the impact on signal preservation. The $\left(\mathrm{f}-\mathrm{k}_{\mathrm{x}}-\mathrm{k}_{\mathrm{y}}\right)$ filter is a robust, well understood technique, which provides, in general, good results in the cross spread domain with computational efficiency. Wavelet transform tools can deliver superior results in some instances, but they are currently less easy to handle in actual projects. They do, however, provide non linear filtering capabilities, and, in some instances, the option of applying the filter in the shot point domain, avoiding computationally expensive cross spread sorting

\section{Introduction}

Source generated coherent noise (surface waves) is probably the most difficult issue to resolve in land data processing, especially when we consider the more stringent requirements for signal preservation in reservoir characterization or time-lapse surveys. The benefit of using an (x-y) spatial sampling for source-generated surface wave filtering is well established. The aim of this work is to compare the results obtained with several coherent noise 3D filtering techniques on real data sets. More specifically, we compare the results obtained through the $3 \mathrm{D}$ extension of the popular (f-k) wavenumber filter, with the results obtained with its time-variant implementation through a wavelet transform. In a second step, we discuss the potential pitfalls which challenge the application of these filtering tools for real 3D acquisition geometries.

\section{D geometries}

In this paper the filters are applied on two kinds of 3D traces ensembles:

- the 3D shot point (SP) geometry,

- the cross-spread (XS) geometry.

3D SP geometries are normally not suitable for 3D filtering because the receivers line spacing is usually so wide that spatial aliasing is significant. However SP geometries can be tested because some filtering methods can deal at least

partially with this difficulty. In addition, sometimes very dense spatial sampling in SP domain is available in desert areas.

To overcome the spatial aliasing issue, the data is usually sorted into cross spread (XS) sub-volumes (Vermeer, 1998). Each XS is built with the traces from one receiver line and one orthogonal shot line. Each trace is binned into an offset / azimuth consistent position, the source-generated surface waves are mapped to a cone, and the data is dealiased because spatial sampling is now controlled by the sources and receivers spacing, This conceptual construction can be adapted to the many types of $3 \mathrm{D}$ shooting practiced in the real world.

\section{D filtering tools}

We compare the results of two kinds of 3D denoise tools, ie the wavenumber (f-k) filter, and wavelet transform filters. The extension of the popular $2 \mathrm{D}(\mathrm{f}-\mathrm{k})$ filter to a $3 \mathrm{D}\left(\mathrm{f}-\mathrm{k}_{\mathrm{x}}-\mathrm{k}_{\mathrm{y}}\right)$ filter is achieved through the cascade of Fourier transforms along each spatial dimension. Filtering is performed by multiplying the 3D Fourier coefficients with a tapered mute template.

The wavelet transform decomposes the signal over dilated and translated versions of a function with compact support (the mother wavelet). Widely used in seismic data compression (Villasenor 1996), their time-scale variant properties are attractive for dispersive noise removal. Several examples of 2D filtering have already been published (Abdul Jauwad 2000, Deighan 1997, Nguyen 2000, Watts 1998). For this work we use two kinds of discrete wavelet transform (Fig 1) with bi-orthogonal filters bases.

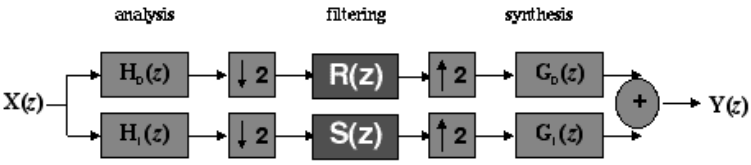

Figure 1 One level wavelet transform decomposition and filtering. Full decomposition is achieved by cascading the $\mathrm{H} 0, \mathrm{H} 1$ filters and downsampling step on the $\mathrm{H} 1$ low pass output. The noise filters $\mathrm{R}$ and $\mathrm{S}$ can be different for each channel.

We used a classical 7-9-tap filter bank which is efficient because it keeps the total number of samples constant by decimation, and we compare with a spline implementation without downsampling because this transform has shift invariance properties. 3D decomposition is performed by cascading the wavelet transform along each time and space dimension. Each channel of the decomposition is actually a 


\section{Practical aspects of 3D coherent noise filtering}

3D volume with its specific time and space spectral content, which can be filtered by any technique.

\section{Comparative results}

We use vibroseis data to make $3 \mathrm{D}$ filter comparisons. The cross-spread domain filtering is carried out on $180 \times 180$ trace volumes providing a spatial sampling of $25 \times 25$ meters. For shot point domain filtering, we use $8 \times 90$ trace volumes with $50 \times 250$ meters spatial sampling.

For the cross-spread case, Figure 2 displays an example of the results of $3 \mathrm{D}$ coherent noise filtering by applying the two kinds of methods. Both techniques achieve a significant level of de-noising. In general a conventional ( $f, k_{x}, k_{y}$ ) filter will be sufficiently robust and easier to tune than a wavelet transform filter. Wavelet transform filters are superior especially when random noise level is high, the data is piecewise continuous (strong static corrections), or when non linear filters are required.

In the shot point domain, we use only the wavelet transform, because we have a limited number of samples in the crossline direction. Fig 3 shows an example of a 3D wavelet transform filter. The collection of traces is first decomposed into 40 sub-bands (each being band-limited in both frequency and wavenumber). A velocity filter and a spatial median filter (band adapted) are applied prior to data reconstruction.

Finally, we study the importance of using shift invariant wavelet transform filters. Duval (2002) discussed some theoretical aspects of this issue. Filtering results are improved when shift invariant filters are used (Fig 4) but the signal representation becomes highly redundant, and the computer efficiency is mostly lost.

\section{D irregularities}

Real data sets seldom sample the data on a regular spatial grid. For example in cross-spreads, besides small deviations from the theoretical trace positions, larger scale irregularities are common (Fig 5), and these irregularities in the data will have an impact on the de-noising and signalpreserving capabilities of the filters. It is possible to carry out data regularization in the temporal frequency domain using irregular Fourier transform techniques (Hindriks 2000), but irregularities, such as gaps or significant departures from a regular bin grid, cannot be compensated easily in the case of strong spatially aliased data.

We measure the impact of large gaps on the filters by carving artificial holes in a pilot cross-spread, and we compute the difference in amplitude with the filtered full volume. As expected, the edge effects found for the wavelet transform filters are narrower because of the local properties of the operators. The trace interpolation work can be restricted to shorter distances in large gaps.

\section{Conclusions}

The $\left(\mathrm{f}-\mathrm{k}_{\mathrm{x}}-\mathrm{k}_{\mathrm{y}}\right)$ filter is currently the most convenient technique if one is looking for a fast and robust 3D velocity filter. Even when the stack fold is very high, it provides a significant additional noise reduction power (Fig 6). However, large sampling irregularities cannot always be compensated, and they do have an impact on signal preservation. Wavelet transform techniques can deliver superior results in this respect, but they are less flexible to use because of their poor sampling (in general) of the velocities. Shift-invariant wavelet transforms provide superior results to decimated techniques, at the cost of greater computational and memory management complexity. When the $\left(\mathrm{f}-\mathrm{k}_{\mathrm{x}}-\mathrm{k}_{\mathrm{y}}\right)$ filter fails to solve the problem, non linear filters with wavelet transform are an alternative, and, in some instances, it is possible to apply the filter in shot point domain, avoiding the sorting of data into cross-spreads.

\section{References}

S. H. Abdul-Jauwad, M. F. Khène, 2000, Two-dimensional wavelet-based ground roll filtering. In Expanded Abstracts, pages 2107-2110, Soc. Expl. Geophys.

A. J. Deighan, D. R .Watts, 1997, Ground-roll suppression using the wavelet transform, Geophysics,62(6):1896-1903.

L. C. Duval, P.-Y. Galibert, 2002, Efficient coherent noise filtering: An application of shift-invariant wavelet denoising. To be published in Expanded Abstracts, $64^{\text {th }}$ EAGE meeting.

K. Hindriks, A. J. W. Duijndam, 2000, Reconstruction of 3D seismic signals irregularly sampled along two spatial coordinates. Geophysics, 65(1):253-263.

Thang Nguyen, J. Castagna, 2000, Matching Pursuit of two dimensional seismic data and its filtering application. In Expanded Abstracts, pages 2067-2069, Soc. Expl. Geophys.

G. Vermeer, 1998, 3-D symmetric sampling. Geophysics, 63(5):1629-1647.

J. D. Villasenor, R. A. Ergas, P. L. Donoho, 1996, Seismic data compression using high dimensional wavelet transforms. In Expanded Abstracts, pages 396-405, Soc. Expl. Geophys.

D. R. Watts, D. K. Smythe, A. J. Deighan, 1998, Removal of airblast from a 3D engineering seismic survey with a wavelet packet filter. In Expanded Abstracts, pages 821824, Soc. Expl. Geophys. 


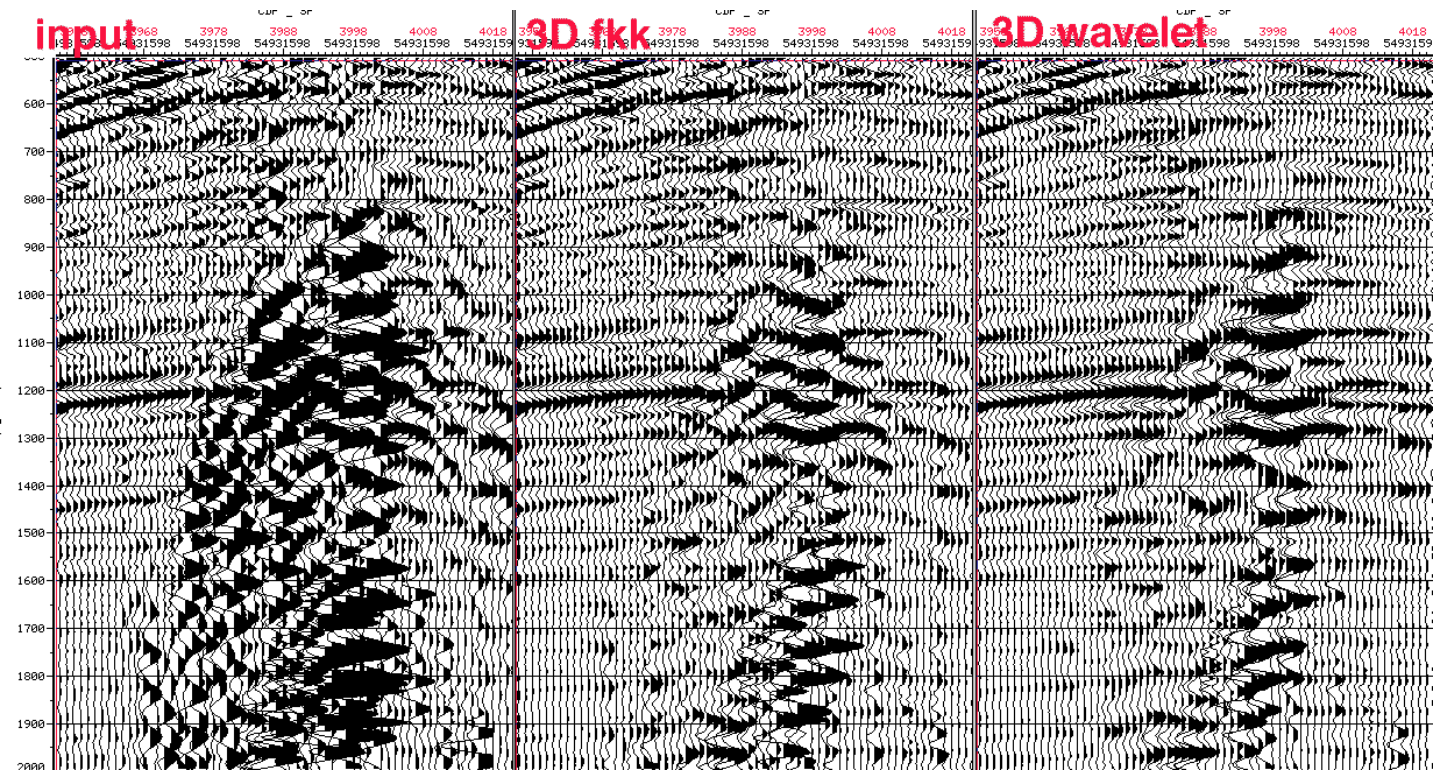

Figure 2 Comparison of 3D filtering in XS domain (part of one receiver line): input (left), ( $\mathrm{f}-\mathrm{k}_{\mathrm{x}}-\mathrm{k}_{\mathrm{y}}$ ) filter (middle), wavelet transform filter (right).

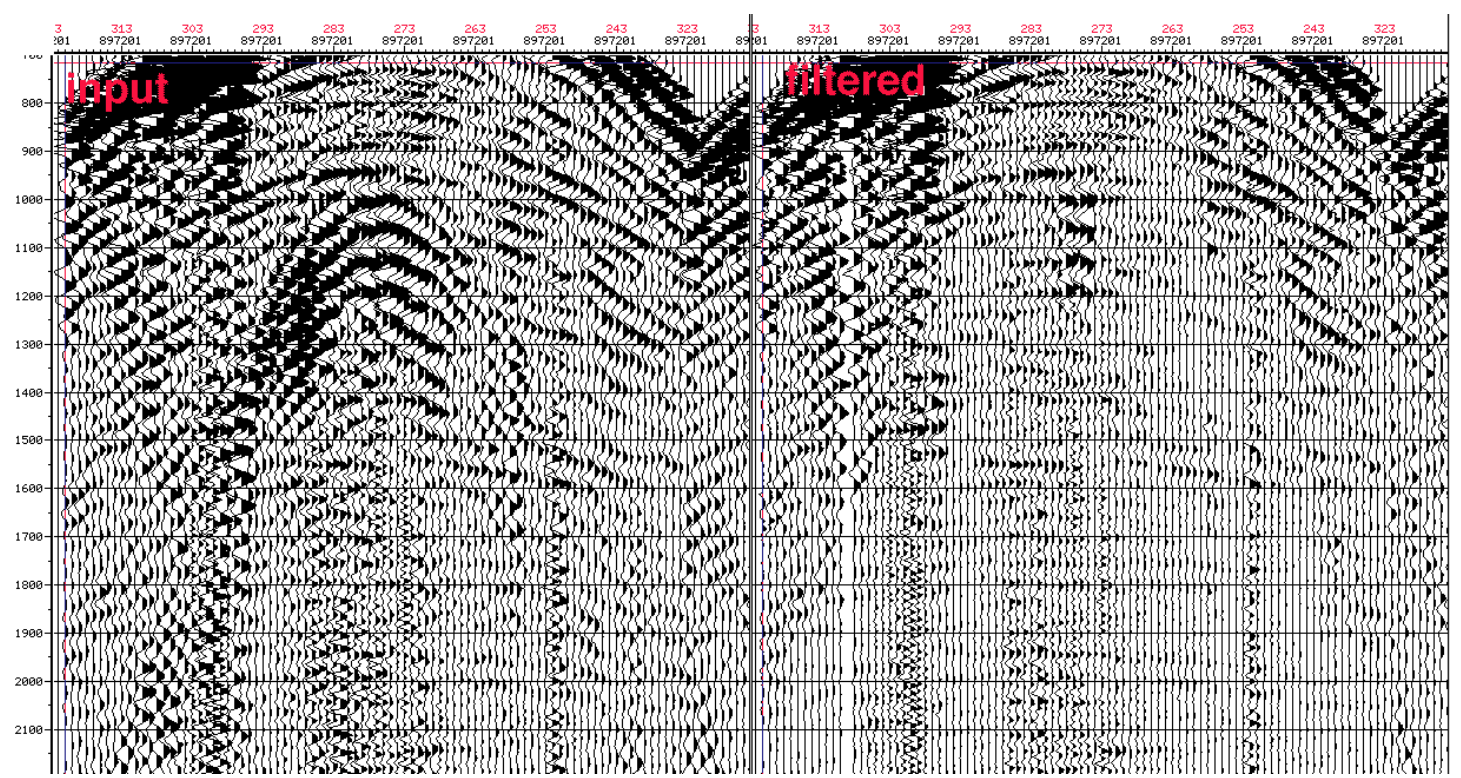

Figure 3 Example of 3D filtering in shot point domain ( $8 \times 90$ receivers) with non linear wavelet transform filter. Part of one receiver line. 


\section{Practical aspects of 3D coherent noise filtering}
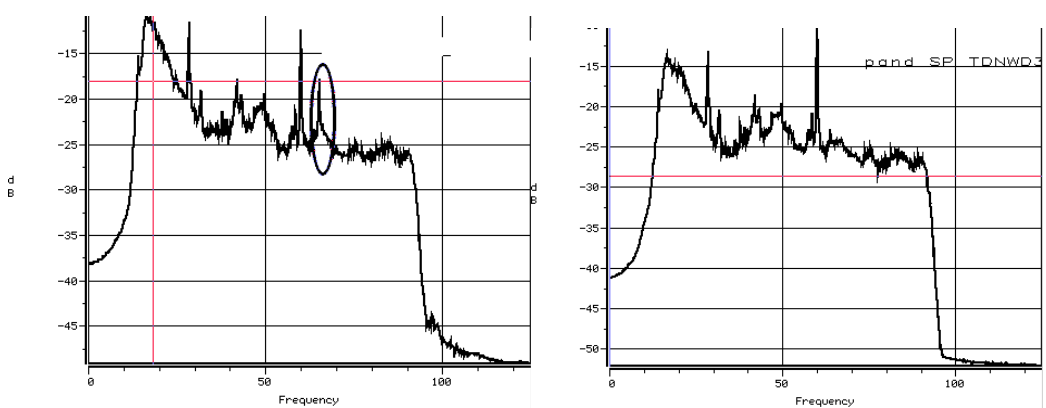

Figure 4 Power spectra of filtered traces using decimated (left) and shift invariant (right) wavelet transform. Note the $65 \mathrm{~Hz}$ artefact (ellipse) generated by multiple frequency folding and wrong unfolding of the aliased $60 \mathrm{~Hz}$.

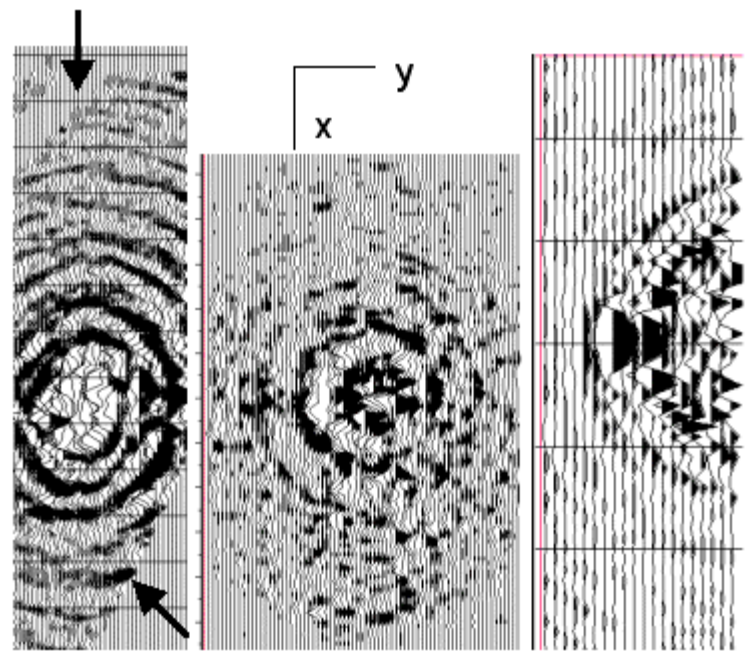

Figure 5 Examples of real XS spatial irregularities of different scales displayed in time slices. Gaps (left), brick pattern (middle), one sided XS (right).
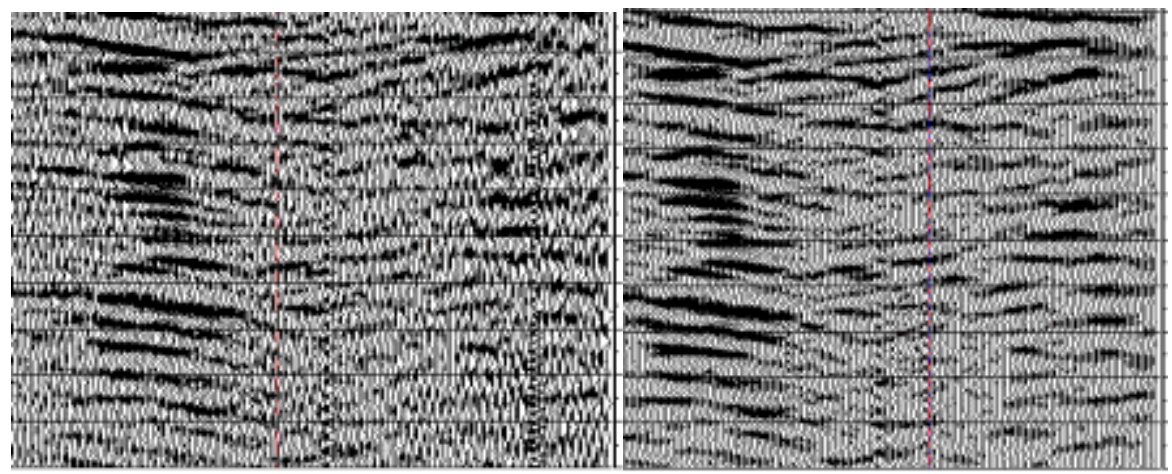

Figure 6 Inline slice in 3D stack: 150 fold stack without 3D fk filter (left). Stack with 3D fk filter (right). 